\title{
¿Cómo se piensa la ciudad? Análisis crítico de un siglo de gestión y planificación urbana
}

$\mathrm{L}$ a sociedad contemporánea se transforma de prisa y, desbordados por la evolución constante, a veces medimos mal cómo han cambiado en poco tiempo los objetos que utilizamos, nuestra forma de actuar, de trabajar, las relaciones familiares, las diversiones, los desplazamientos, las ciudades en las que vivimos. Sin lugar a dudas, esta dificultad para percibir los cambios también es observable en el ámbito del desarrollo urbano. No obstante, numerosos indicios y análisis nos llevan a pensar que constantemente "se hacen necesarios cambios importantes en el concepto, la producción y la gestión de las ciudades y de los territorios" (Ascher, 2004: 17-18).

Entendiendo que el análisis crítico de los procesos históricos permite la aprehensión de aspectos que marcan las disciplinas en este enlace dialéctico del presente y del pasado (Monteiro y Silva, 1994), el siguiente escrito se plantea como objetivo una revisión de aquel proceso que desencadena el cambio entre un modelo holístico-normativo-centralista de gestión urbana a aquel que se adecúa estratégicamente a las nuevas exigencias territoriales. Todo esto, sin perder nunca de vista la comprensión y proyección de lo que vendrá a futuro.

Para ello, se plantea que el proceso de transformación de la gestión urbana se instala en uno bastante más amplio, denominado modernidad, el cual persigue, muy esencialmente, la creación de una sociedad racional. Según Touraine (1994; 18), "la modernidad ha hecho de la racionalización el único principio de organización de la vida personal y colectiva al asociarlo al tema de la secularización, es decir, prescindiendo de toda definición de los 'fines últimos".
En este sentido, se entiende que si bien la modernidad no es un estado, la modernización tampoco es un proceso continuo, y es posible distinguir, hasta ahora, tres grandes fases: Edad Moderna ${ }^{1}$, Revolución Industrial y Modernidad Radical ${ }^{2}$ (Ascher, 2004). Para efectos de este texto, sólo se abordarán las dos etapas más recientes del proceso moderno. Como es de suponerse, a cada una de ellas le corresponden principios y modos de concepción y organización del territorio más o menos específicos, los cuales se analizan a continuación.

\section{La segunda revolución urbana moderna}

Este período, correspondiente a la Revolución Industrial, "comenzó con la revolución agrícola -que incrementó la producción de alimentos pero expulsó del campo a gran cantidad de agricultoresy con el desarrollo concomitante del capitalismo industrial. Este doble proceso provocó un enorme crecimiento demográfico en las ciudades, lo que supuso una expansión espacial acelerada que dio lugar, al mismo tiempo, a una grave pauperización de una parte de las poblaciones urbanas" (Ascher, 2004: 24-25).

Para algunos grupos, el advenimiento de la era maquinista había provocado la entrada del caos a las ciudades, ante lo cual estas ciudades se desviaban absolutamente de su "destino", que sería el satisfacer las necesidades biológicas y psicológicas primordia-

\footnotetext{
Va desde el fin de la Edad Media hasta el principio de la Revolución Industrial.

Entre otras denominaciones, como se verá más adelante.
} 
les de sus habitantes. La causa, se decía, estaba en la ausencia de reglas lógicas con las cuales someter el florecimiento industrial: "Al contrario, todo ha sido abandonado a la improvisación, que, si alguna vez favorece al individuo, agobia siempre a la colectividad" (CIAM, 1957: 87).

Estas aseveraciones provenían de la convicción de que el ser humano poseía la capacidad para poder conducir racionalmente los procesos sociales (De Mattos, 2005). En este sentido, el éxito de algunas teorías científicas, y en particular la teoría de la gravedad de Newton, llevaron a argumentar, a principios del siglo XIX, que el universo era completamente determinista. Laplace, científico francés, "sugirió que debía existir un conjunto de leyes científicas que nos permitirían predecir todo lo que sucediera en el universo, con la única condición de que conociéramos perfectamente su estado en un momento determinado" (Fernández Güell, 1997: 58).

Si bien la doctrina del determinismo científico fue ampliamente criticada por diversos sectores, por considerar que infringía la libertad divina de intervenir en el mundo, se constituyó como el paradigma de la ciencia, la cultura y la economía hasta los primeros años del siglo pasado (Fernández Güell, 1997). Es así como, durante la primera mitad del siglo XX, el fordismo, sistema de producción imperante en la época, basaba su funcionamiento en la previsibilidad del futuro. Las empresas podían producir antes de vender, amortizar las variaciones con los stocks e invertir a largo plazo. En este contexto, "la planificación era uno de los instrumentos fundamentales para los países, las empresas, para el desarrollo urbano y la ordenación territorial" (Ascher, 2004: 45-46).

Por lo tanto, no debería sorprender que el urbanismo moderno también se haya propuesto corregir las ciudades que hacían la desgracia del hombre

* Instituto de Estudios Urbanos y Territoriales, Pontificia Universidad Católica de Chile. E-mail: lparragu@uc.cl

** Instituto de Estudios Urbanos y Territoriales, Pontificia Universidad Católica de Chile. E-mail: mgrodri1@uc.cl

*** Instituto de Estudios Urbanos y Territoriales, Pontificia Universidad Católica de Chile. E-mail: mbsantan@uc.cl
(CIAM, 1957) a través de diversas propuestas también representativas de un racionalismo radicalizado. Ellas "expresaban la convicción de los planificadores urbanos sobre su plena capacidad para modelar y estructurar a las ciudades conforme a planes directores representativos de un urbanismo arquitectónico (De Mattos, 2003). Un ejemplo paradigmático de esta visión fueron las propuestas de Le Corbusier en respuesta al caos en las ciudades, que culminaron en la Carta de Atenas. Esta Carta, "puesta en manos de la autoridad, detallada, comentada, iluminada por una explicación suficiente, [era] el instrumento por el cual ser[ía] enderezado en destino de las ciudades" (CIAM, 1957: 30).

Como puede observarse a través de este ejemplo, la gestión y la planificación urbana de este período también dejan entrever un marcado carácter centralizado. Es necesario mencionar que los poderes públicos, en el marco del desarrollo del Estado de Bienestar, se habían visto abocados a actuar cada vez más en el campo del urbanismo, especialmente para hacer frente a las insuficiencias, incoherencias y "disfunciones" de las lógicas privadas y de los mercados, en particular, en los aspectos territoriales e inmobiliarios. De esta forma, desde el Estado se crean "todo tipo de estructuras y procedimientos para 'planificar' de forma más racional las ciudades, es decir, lo más científicamente posible, para actuar a pesar de las coacciones de la propiedad privada" (Ascher, 2004: 26).

Es el caso de la U.R.S.S., donde el Estado socialista, al conocer y tener en cuenta la acción de las leyes económicas, dirige planificadamente el desarrollo de la economía nacional. Según Kadishev y Sorokin (1970), las leyes económicas del socialismo existían independientemente de la voluntad y la conciencia de las personas, pero ello no implicaba, ni mucho menos, espontaneidad en el desarrollo de la economía de esa nación. Es más, "el carácter estatal de la planificación da a esta última un carácter de directriz. Son precisamente las decisiones estatales las que, ante todo, hacen que las tareas del plan sean obligatorias para todos" (Kadishev y Sorokin, 1970: 90).

Como puede vislumbrarse, se impone una concepción holística de planificación, en donde el valor supremo reside en la sociedad como un todo 
(Dumont, 1987). En este sentido, la Carta de Atenas estableció que la ley que condujese la planificación debería favorecer todas las iniciativas justamente medidas, pero cuidando que se inserten en el plan general y estén siempre subordinadas a los intereses colectivos que forman el bien público. Se determinaba la sabiduría de un plan en la medida que permitía la colaboración fructuosa, al mismo tiempo de cuidar y respetar al máximo la libertad individual (CIAM, 1957).

\section{El quiebre del modelo predictivo}

Como se ha expuesto hasta ahora, la planificación tradicional o de la Edad Moderna estaba basada esencialmente en predicciones, las cuales funcionaron razonablemente bien durante las décadas "estables" de los '50 y '60. Sin embargo, desde principios de los '70 los errores de predicción han llegado a ser más frecuentes y, en ocasiones, de una magnitud dramática y sin precedentes (Fernández Güell, 1997).

Uno de los principales puntos de quiebre fue la crisis progresiva del sistema fordista a finales de los años sesenta: la producción masiva chocó con la diferenciación social y la diversificación de la demanda. Las tecnologías y las formas de organización que habían garantizado el crecimiento de la producción y la productividad llegaron a su límite. Las recetas keynesianas se volvieron antiproductivas en economías más abiertas, la intervención del Estado de Bienestar se volvió muy cara y tuvo efectos perniciosos (Ascher, 2004). Específicamente, el Estado sufría una crisis fiscal debido a la contradicción entre los gastos crecientes (determinados por las demandas sociales) y los ingresos comparativamente decrecientes (limitados por la necesidad de mantener el nivel de beneficios de las grandes empresas) (Castells, 1995).

En suma, la crisis del sistema en los años '70 reveló la falta de efectividad de los mecanismos establecidos en los años ' 30 y ‘ 40 para asegurar la satisfacción de las metas básicas de la economía capitalista (Castells, 1995), lo cual aumenta la incertidumbre y sienta las bases para el nacimiento de una nueva forma de economía de mercado (Ascher, 2004).

\section{La tercera revolución urbana moderna}

Es precisamente en este escenario donde se plantea que entramos en una tercera fase o episodio de la modernización, que algunos autores han calificado de modernidad "radical", modernidad "avanzada", "sobremodernidad” o "baja modernidad". Se subraya "el hecho de que la sociedad moderna se separa de un racionalismo demasiado simplista y de sus certezas, y se desprende de formas de pensamiento mesiánicas o providenciales que aún marcan la idea moderna de progreso" (Ascher, 2004: 30).

Así, se produce la adopción generalizada de un "nuevo saber convencional dominante", asociado a la liberalización económica, a una revalorización del papel del mercado y a la recuperación del protagonismo del capital privado en la dinámica de acumulación y crecimiento (De Mattos, 2005). Los cambios económicos en curso ponen de manifiesto que "las sociedades occidentales empiezan a salir del industrialismo, y que están entrando en una economía cognitiva, basada en la producción, apropiación, venta y uso de conocimientos, información y procedimientos" (Ascher, 2004: 44).

En su esencia, el nuevo modelo de desarrollo que se impone paulatinamente durante los años ' 80 tiende a reforzar el carácter estrictamente capitalista de la lógica económica, imponiendo más rigurosamente la exigencia de rentabilidad de las inversiones como principio regulador de la economía (De Mattos, 2003). Este proceso ha exigido la integración progresiva de las naciones-Estado en bloques continentales y la apertura de los mercados comerciales a nivel global, lo cual ha dado lugar a una abierta rivalidad entre ciudades para captar inversiones, puestos de trabajo, visitantes y ayudas públicas (Fernández Güell, 1997).

Una de las principales razones de ello dice relación con que los diversos agentes sociales y económicos, que tradicionalmente han actuado en la ciudad, han comenzado a pedir con insistencia el cumplimiento de una serie de requerimientos de competitividad y habitabilidad como condición para su permanencia en un área urbana determinada, lo cual ha obligado a los gestores públicos a considerar las exigencias de dichos agentes y a contar con ellos en el momento de tomar decisiones. Esto les demanda una gran capacidad de anticipación y, en su defecto, de reacción ante las actuaciones estratégicas de sus competidores más directos (Fernández Güell, 1997). 
Todos estos procesos, como veremos, producen implicaciones de gran magnitud para el desarrollo urbano, las cuales, a su vez, obligan a la transformación y renovación de los instrumentos tradicionales de planificación. Tanto a nivel teórico como desde la práctica gubernamental, se llega generalizadamente a la conclusión de que una planificación centralizada, normativa y basada en una racionalidad sustantiva, como la que estuvo asociada a la "planificación de desarrollo económico y social” y a todas sus derivaciones, son inaplicables y carecen de toda operatividad en este tipo de sociedad (De Mattos, 2003).

Como se ha visto, "el futuro había pasado de ser un objeto relativamente estable a convertirse en un objeto volátil [...]. Por esta razón, los planificadores contemporáneos se enfrentan a demasiadas fuerzas que obstaculizan la posibilidad de alcanzar predicciones correctas" (Fernández Güell, 1997: 58). Ante esta situación, la postura más inteligente es aceptar la incertidumbre, tratar de comprenderla y convertirla en parte de nuestro razonamiento: "En el momento presente, la incertidumbre no es sólo una desviación ocasional y temporal respecto a una predicción razonable, sino que es una faceta estructural del entorno socioeconómico. Por lo tanto, resulta obvia la inconveniencia de aplicar modelos evolutivos a largo plazo que pretendan proyectar con precisión el futuro del desarrollo urbano [...]. En su lugar, se requieren herramientas de análisis que ofrezcan mayor flexibilidad en la comprensión de un entorno cada vez más dinámico y complejo" (Fernández Güell, 1997: 5859).

De esta manera, la tercera revolución urbana moderna-que se inicia con la nueva fase de modernización de las sociedades occidentales-suscita cambios profundos en las formas de pensar, construir y gestionar las ciudades. Surge el llamado neourbanismo, el cual se apoya en una gestión más reflexiva, adaptada a una sociedad compleja y a un futuro incierto, propio de una sociedad abierta, democrática y marcada por la aceleración de la nueva economía (Ascher, 2004).

Y como principal instrumento de planeación de ciudades surge la Planificación Estratégica. Este concepto es extraído de la práctica militar, el cual comenzó a utilizarse como instrumento analítico y de- cisorio en el mundo empresarial a partir de la segunda mitad del siglo pasado. Luego se extiende a la gestión pública y actualmente se aplica también al campo de la planificación y gestión urbana (Fernández Güell, 1997).

Consiste básicamente en un proceso creativo que sienta las bases de una actuación integrada a largo plazo, estableciendo un sistema continuo de toma de decisiones que comporta riesgo, identifica cursos de acción específicos, formula indicadores de seguimiento sobre resultados e involucra a los agentes sociales y económicos locales a lo largo de todo el proceso (De Mattos, 2003). Como puede observarse, este nuevo instrumento viene a revolucionar la antigua cronología lineal ${ }^{3}$ de planificación, "por una gestión heurística ${ }^{4}$, iterativa ${ }^{5}$, incremental ${ }^{6}$ y recurrente $^{7}$, es decir, por actos que sirven al mismo tiempo para elaborar y probar hipótesis, con realizaciones parciales y medidas a largo plazo que modifican el proyecto y la retroalimentación tras las evaluaciones y que se traducen en la redefinición de los elementos estratégicos" (Ascher, 2004: 73).

En el actual paisaje socioeconómico, no debería sorprender que esta nueva manera de gestionar ciudades se ponga al servicio de un enfoque productivista, guiado por la búsqueda del crecimiento y la competitividad con un énfasis manifiesto en la atracción de inversiones y actividades generadoras de empleo (Rodríguez et al., 2001). En este sentido, "los nuevos modelos han llevado a introducir la idea de gestión endógena como medio de activar el potencial de cada territorio (nacional o subnacional), y de esta manera estimular su crecimiento. En este enfoque subyace la consideración de que, en el ámbito de una economía globalizada, el objetivo básico

Diagnóstico, identificación de necesidades, elaboración final de un plan, programación, proyecto, realización y gestión.

Que sirve para el descubrimiento, que procede por evaluaciones sucesivas e hipótesis provisionales.

Método de resolución de una ecuación por aproximaciones sucesivas.

6 Cantidad en la que se aumenta una variable en cada ciclo de un bucle de un programa.

Serie recurrente: aquella en la que cada uno de sus términos es una función de los términos inmediatamente anteriores. 
de una gestión endógena debería ser aumentar la competitividad de los productos nacionales, regionales o locales, de manera de maximizar sus posibilidades exógenas" (De Mattos, 2003: 27).

A partir de estos procesos, algunos autores se atreven a adelantar frecuentemente la hipótesis de una próxima desaparición del Estado soberano clásico, hipótesis fundada sobre la noción del territorio e internacionalización de las actividades económicas en un mundo cada vez más desprovisto de fronteras. En este sentido, aparece un nuevo paradigma: el desarrollo "desde abajo" o desarrollo local, que reemplaza al desarrollo "desde arriba" administrado por el Estado (Benko, 2000).

La importancia de la estrategia de lo local como centro de gestión de lo global en el nuevo sistema tecno-económico puede apreciarse en tres ámbitos principales: el de la productividad y competitividad económica; el de la integración sociocultural, y el de la representación y gestión políticas (Borja y Castells, 1996). Respecto a este último punto, surge el concepto de gobernanza como sistema de gobierno que permite articular y asociar las instituciones políticas, los actores sociales y las organizaciones privadas locales en torno a objetivos propios, discutidos y definidos colectivamente en ámbitos fragmentados e inciertos (De Mattos, 2005).

En el nuevo contexto de competitividad entre ciudades, las políticas locales y sus procesos de gestión se fijan como objetivo principal la promoción del crecimiento económico de cada sistema productivo local, sea cual fuera su dimensión (De Mattos, y 2005; Rodríguez et al., 2001). Las estratégicas de revitalización urbana no son sólo la punta de lanza de las políticas urbanas, sino la expresión material de una reorientación radical de la agenda política de las ciudades (Rodríguez et al., 2001).

Esta reorientación estratégica exige proyectar una imagen de ciudad dinámica e innovadora, estimulante y creativa, capaz de competir con éxito por la atracción tanto de inversiones productivas y funciones direccionales como consumidores internacionales (Rodríguez et al., 2001). Hoy día, por tanto, las ciudades deben hacer su propia promoción.

Según Benko (2000), esto no constituye un fenómeno fundamentalmente nuevo, ya que la liber- tad de mercados (laboral, de bienes, servicios y de capitales) siempre ha incitado a las ciudades a "cuidar su imagen”, pero la aceleración de los procesos económicos ha incrementado verdaderamente las necesidades de nuevas herramientas que apuntan a aumentar su atractivo. Como consecuencia, el marketing territorial se ha convertido en una realidad de la vida económica, política y social, incluso alterando la representación espacial e influyendo en nuestra percepción de la realidad geográfica (Benko, 2000).

\section{Conclusiones}

Teniendo en claro los principales elementos que involucra la adopción de la Planificación Estratégica como instrumento estrella de la gestión urbana contemporánea, ¿es posible definir su real efectividad? ¿Cuáles serían los principales problemas que acarrea para los distintos territorios? Mucho podría decirse al respecto, pero primordialmente se ilumina el hecho de que "la mayor parte de los planes estratégicos elaborados hasta la fecha han puesto un énfasis, quizá excesivo, en los aspectos de competitividad económica y no se han esforzado por explorar la vía de desarrollo sostenible” (Fernández Güell, 1997: 13). Por lo tanto, como puede comprobarse fácilmente, un particularismo mal entendido ha generado una competición excesiva y destructiva entre distintas localidades y regiones (Borja y Castells, 1996).

Ante este hecho, se abre la convicción de que ya no es posible entender los cambios en el modelo de gestión urbana como el simple paso de una visión tradicional a una moderna. En nuestros territorios, y también al interior de sus ciudades, con expresiones diferenciadas, vivimos un proceso de doble rostro en un tiempo de capitalismo mundialmente integrado. Por una parte, hay exigencias crecientes de transnacionalización, de competencia segmentada. Por otra, esta explosión de demandas, criterios normas hacen que las formas de marginalidad se diversifiquen y acentúen. La dialéctica de la modernización consiste precisamente en esta contradicción (Matus, 1999).

Por lo tanto, la planificación estratégica como instrumento no es ni pretende ser interpretada como la panacea que dé respuesta a todos los problemas que lleva aparejada la planificación y gestión urbana. La generación de unas expectativas desmesura- 
das sobre los posibles resultados de estos procesos ha dado lugar a frustraciones que han podido restarles credibilidad.

Para revertir esta situación, se debería entender que todo proceso de planificación urbana para obtener real efectividad debe ser integrado coherentemente dentro de procesos de planificación social y gubernamental mucho más amplios (Fernández Güell, 1997). En ellos, el principal desafío futuro deberá ser la búsqueda de caminos que permitan compatibilizar los objetivos de competitividad de naciones, regiones y ciudades, con los de mejoramiento de las condiciones de vida de los habitantes de esos ámbitos (De Mattos, 2005). Específicamente deberían plantearse, entre otros aspectos, la construcción de redes cooperativas y solidarias entre ciudades y regiones, lo cual les permita negociar constructivamente con las empresas para alcanzar acuerdos de interior común, y la gestión de las diferencias socioculturales de los distintos grupos de población que cohabitan un espacio y su integración en una cultura compartida que no niegue las especificidades históricas, culturales y religiosas (Borja y Castells, 1996).

Por lo tanto, el análisis crítico de los procesos que conllevan a la transformación histórica de la gestión urbana nos lleva a apreciar que, al igual que la modernidad, enfrenta un proceso incompleto. $\mathrm{O}$ mejor dicho, se encuentra frente a una elección. Puede someterse enteramente a la lógica de la acción instrumental y de demanda mercantil o combinar razón y sujeto, eficacia y libertad. Combinación cargada de conflictos, pero conflictos entre fuerzas que comparten la misma referencia a la creatividad humana y al repudio de todas las esencias y todos los principios de orden.

\section{Referencias bibliográficas}

Ascher, F. (2004). Los nuevos principios del urbanismo: el fin de las ciudades no está a la orden del día. Madrid: Alianza.

Benko, G. (2000). "Estrategias de comunicación y marketing urbano". EURE Revista Latinoamericana de Estudios Urbano Regionales, 26, 79.

Borja, J. y M. Castells (1996). Local y global. La gestión de las ciudades en la era de la información. Madrid: Taurus.

Castells, M. (1995). La ciudad informacional. Tecnología de la información, reestructuración económica y el proceso urbano-regional. Madrid: Alianza.

Congresos Internacionales de Arquitectura Moderna (CIAM) (1957). La Carta de Atenas. Buenos Aires: Contémpora.

De Mattos, C. (2003). De la planificación a la governance: implicancias para la gestión urbanoregional (mimeo). (2005). De la planificación a la gobernanza: nuevos rumbos en gestión urbana (mimeo).

Dumont, L. (1987). Ensayos sobre el individualismo: una perspectiva antropológica sobre la idolología moderna. Madrid: Alianza.

Fernández Güell, J. M. (1997). Planificación estratégica de ciudades. Barcelona: Gustavo Gili.

Kadishev, L. y G. Sorokin (1970). Leyes económicasy planificación socialista. México: Grijalbo.

Matus, T. (1999). "Los requisitos de una intervención social fundada”. Propuestas contemporáneas de trabajo social. Buenos Aires: Editorial Espacio.

Monteiro, R. y L. M. da Silva (1994). "Historia personal e historia social". Revista de Trabajo Social, 62.

Rodríguez, A., F. Moulaert y E. Swyngedouw (2001). "Nuevas políticas urbanas para la revitalización de las ciudades en Europa". Ciudady Territorio. Estudios Territoriales, 33, 129.

Touraine, A. (1994). Critica de la modernidad. Buenos Aires: Fondo de Cultura Económica. 